\section{Observing implantable collamer lens dislocation by panoramic ultrasound biomicroscopy}

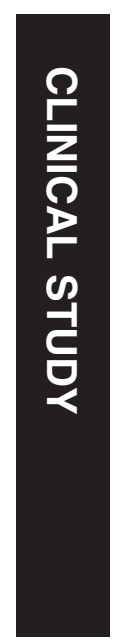

foldable phakic intraocular lens designed to be placed in the posterior chamber to correct high myopia. Some postoperative complications such as cataract and secondary glaucoma closely correlate with the ICL position. Therefore the stability of the ICL position after surgery becomes the major concern in subjects undergoing ICL implantation. Notably, information regarding spontaneous abnormal positioning after ICL implantation is scarce. The purpose of this study is to observe ICL positional changes after ICL implantation in high myopic eyes, capturing images with panoramic ultrasound biomicroscopy (UBM) at various postoperative periods to characterize ICL decentration and dislocation.

\section{Materials and methods}

A total of 127 patients (242 eyes); 64 females $(50.3 \%)$ and 63 males $(49.7 \%)$ were included in this retrospective study. Inclusion criteria for ICL implantation consist of corrected distance visual acuity of $30 / 50$ or better; the meanpreoperative spherical equivalent $-9.08 \pm 2.04$ diopters (D), and the central anterior chamber depth $(A C D) \geq 2.8 \mathrm{~mm}^{1}$. All eyes were implanted with the myopic ICL (ICMV4 model) at the Eye Hospital of China Medical University (Shenyang, Liaoning, China) from July 2007 to September 2011. All subjects underwent preoperative and postoperative panoramic UBM (MD-300L, MEDA Co., Ltd. Tianjin, China) examination to capture images in the superior, inferior, nasal, and temporal directions at various periods of follow-up (1 week preoperative, 1, 3, and 6 months, and
Department of

Ophthalmology, The Fourth Affiliated Hospital of China Medical University, Eye Hospital of China Medical University, The Key Laboratory of Lens in Liaoning Province, Shenyang, China

Correspondence: M Shi, Department of Ophthalmology, The Fourth Affiliated Hospital of China Medical University, Eye Hospital of China Medical University, The Key Laboratory of Lens in Liaoning Province, No.11 Xinhua Road, HePing District, Shenyang, Liaoning 110005, China Tel: +86 1388914 2002; Fax: +86 2423528875 . E-mail:xm5788@163.com

Received: 24 July 2014 Accepted in revised form: 9 December 2014 Published online: 23 January 2015 
yearly postoperatively). Postoperative observation time ranged from 1 month to 36 months, with a mean of $13.67 \pm 13.72$ months. During preoperative appointments, all subjects were fully informed of the details and possible risks of the examination and surgical procedure. Written informed consent was obtained from all patients before examination and surgery following the tenets of the Declaration of Helsinki Principles.

Postoperative observed metrics included central ACD, peripheral anterior chamber angle morphology, the central vault, the anterior capsule of lens, the distance between ICL footplate and lens equator. All image acquisitions were completed by the same physician. The central vault was defined as the distance between the back of the surface of the ICL and the anterior crystalline lens pole (Figure 1c).

\section{ICL size calculation}

The ICL diameter was individually determined based on the corneal horizontal white-to-white distance (WTW) and ACD measured with IOL-Master (ce0297, Carl Zeiss Meditec, Jena, Germany) following the manufacturer's recommendations by adding 0.5 or $1.0 \mathrm{~mm}$ to WTW depending on the ACD.

\section{Surgical technique}

Laser YAG peripheral iridotomy were placed 1 week prior to lens implantation. Cycloplegic eye drops were instilled $30 \mathrm{~min}$ before the surgery to maintain mydriasis during surgery. All surgeries were performed by one experienced surgeon. A primary incision was placed on the steep axis using a 3.0-mm keratome blade. The anterior chamber was filled with sodium hyaluronate $1 \%$ (Provisc; Alcon Laboratories Inc, Fort Worth, TX, USA), which was used to protect the corneal endothelium and lens during ICL implantation using the ICL cartridge and injector (STAAR Surgical). Automated irrigation and aspiration was used to remove the viscoelastics following placement of the ICL into the ciliary sulcus. The miotic was injected into anterior chamber at the end of surgery. No intraoperative complications such as laceration of the anterior lens capsule, placement of an inverted ICL, or ICL decentration were identified.

\section{Results}

The normal anterior segment UBM images of preoperative and postoperative were shown in Figure 1a-d.The preoperative and postoperative mean ACD was $3.27 \pm 0.24 \mathrm{~mm}$ and $2.38 \pm 0.38 \mathrm{~mm}$ respectively. Four ICL footplates were located in the ciliary processes
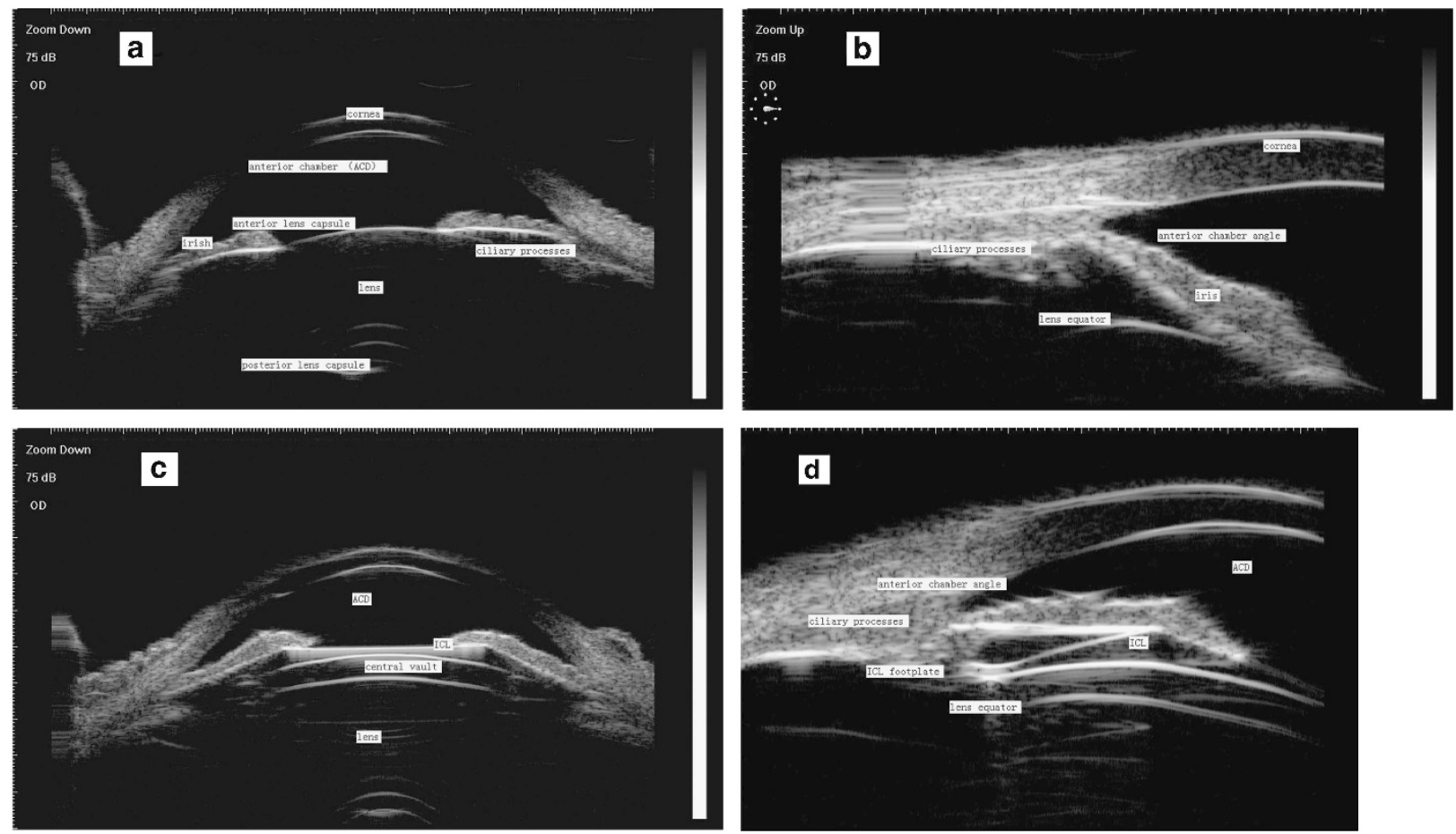

Figure 1 Images of normal preoperative and postoperative anterior segments. Normal preoperative anterior segment. (a) axial section (b) longitudinal section. Normal postoperative anterior segment. (c) axial section and (d) longitudinal section. Figures $2-4$ show the abnormal postoperative anterior segment images. 
where effective distance between the ICL and lens was maintained in each direction (Figure 1c and d). Both smooth arc echoes of ICL back surface and the anterior lens capsule remained almost parallel. Mean postoperatively normal central vault was $0.54 \pm 0.28 \mathrm{~mm}$. Twenty-eight eyes (11.2\%) implanted with ICL had abnormal postoperative positioning. Three groups were classified based on the UBM image characteristics: 12 eyes $(5.0 \%)$ had high central vault anomalies (Figure 2), excessive mean vault is $1.14 \pm 0.39 \mathrm{~mm}$; 10 eyes $(4.1 \%)$ had low central vault anomalies (Figure 3 ) insufficient mean vault is $0.13 \pm 0.11 \mathrm{~mm}$; the remainder $(2.5 \%)$ had ICL decentration without central vault anomalies (Figure 4).

In this study, and in panoramic UBM imaging, ICL decentration appears in one side of the ICL footplate located in the ciliary sulcus, as well as another in the ciliary processes or the back of iris, where the vault was significantly different in every diagonal direction, the opening degree of anterior chamber angle exhibited inconsistencies in each direction,, the most narrow point was about only $12^{\circ}$ (Figures 2 and 4 ). Iris lost its normal arc shape and became straight because of being ejected by ICL and closed to cornea extremely. Another ICL dislocation was the distance between ICL and lens, where the central vault may disappear and the ICL posterior surface was affixed with the anterior crystalline lens (Figure 3).
Figure 1a-d shows UBM images of normal preoperative and postoperative anterior segments.

Figures 2-4 show UBM images of abnormal postoperative anterior segment. ICL abnormal position emerged in 1 month postoperative. Two eyes developed a cataract after ICL implantation 4 and 5 years,

respectively, and were underwent surgery. The elevated intraocular pressure (IOP) occurred in seven eyes with excessive vaults and the occurrence time was ranged from 3 weeks to 6 months after ICL implantation, the mean IOP was $22.12 \pm 3.12 \mathrm{~mm} \mathrm{Hg}$. Among them, three eyes underwent ICL replacement because of the elevated IOP of more than $25 \mathrm{~mm} \mathrm{Hg}$ for 3 months.

\section{Discussion}

The central vault concept defined as the distance between the back of the surface of the ICL and the anterior crystalline lens pole. Several authors previously defined a vault into three groups based on the vault value: an insufficient vault was defined as $<250 \mu \mathrm{m}$, the ideal vault as $250-750 \mu \mathrm{m}$, and excessive vault as $>750 \mu \mathrm{m} \cdot{ }^{2-4}$ An insufficient vault has been identified as a factor that increases the risk of cataract development because of the ICL-crystalline lens contact or interferences with lens nutrition. ${ }^{5-8}$ In this study, 10 eyes had insufficient vault, among them, 2 eyes in two subjects'
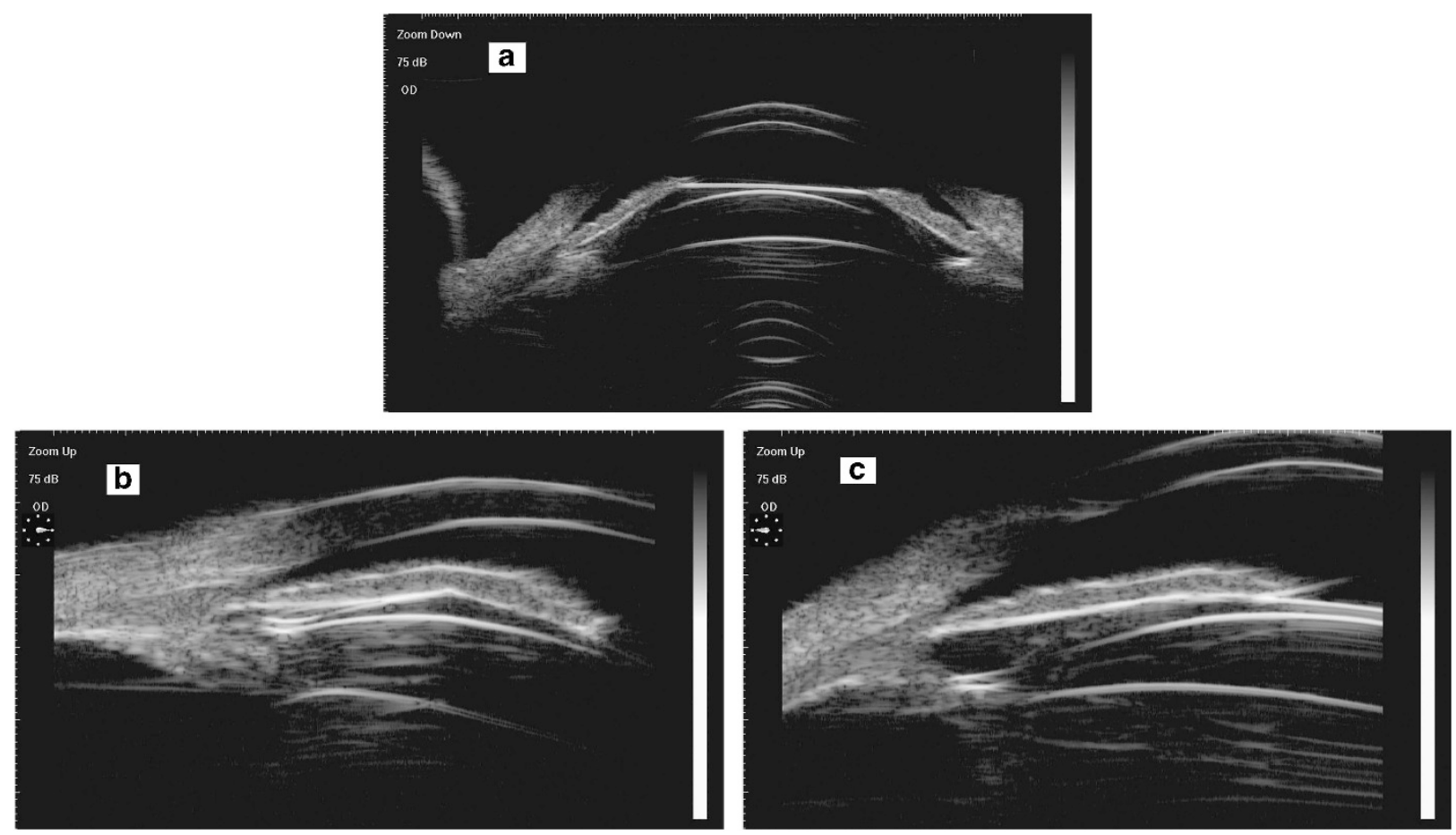

Figure 2 Postoperative excessive central vault (a), one ICL footplate is located in the ciliary sulcus at the 3 o'clock position (b), the other one is located in the ciliary processes at the 9 o'clock position (c). Iris lost its normal arc and became straight because of being ejected by ICL and closed to cornea. 

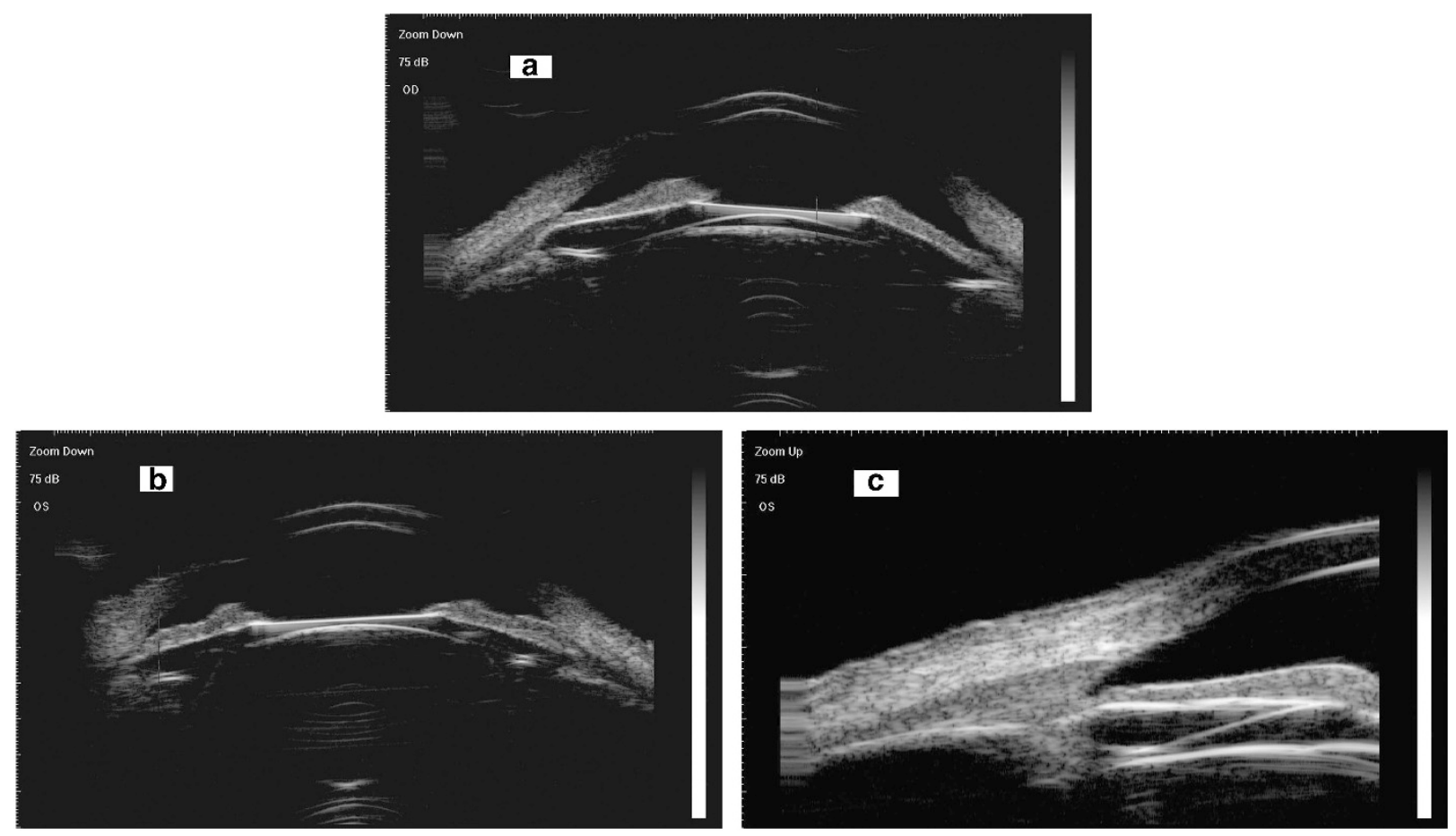

Figure 3 Postoperative insufficient central vault (a) or disappeared (b) In the axial section, the posterior surface of the ICL and the anterior crystalline lens are affixed together, with a mild-subcapsular cataracts evident by the echo of anterior lens capsule (a,b). In the longitudinal section, part of the ICL posterior surface and its loop are attached together to the periphery of the lens (c).
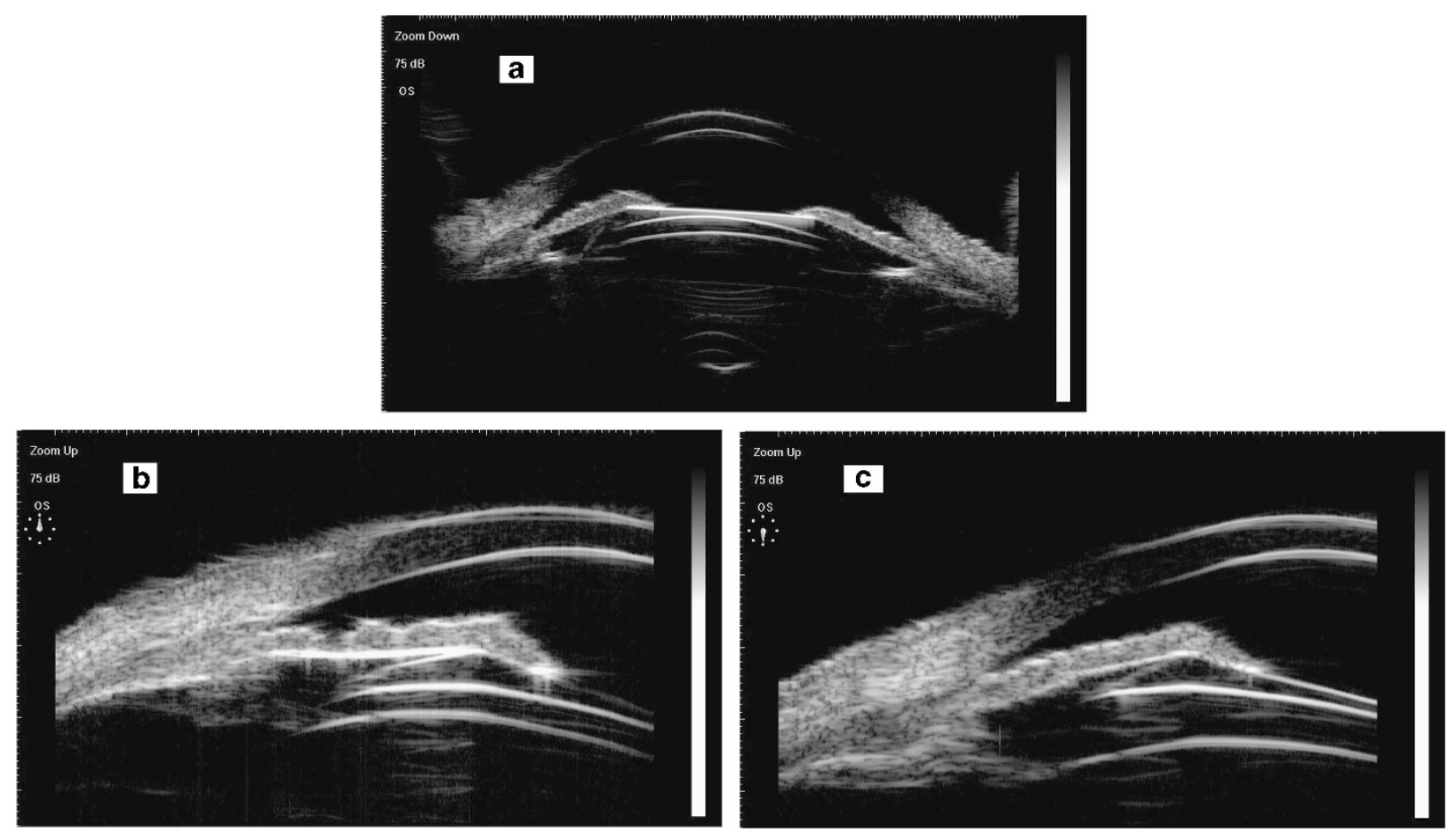

Figure 4 Normal postoperative central vault (a), the distance between ICL and lens equator has inconsistencies. The superior section has a relatively close distance (b), whereas the inferior has a relatively far distance (c). 
ICL-crystalline contact developed a cataract and underwent surgery. In addition, in three eyes in two different subjects the optical centers of their ICL were not in contact with the central anterior lens capsule, but the posterior ICL surface was connected with the lens equator. The point-shape echo under the lens capsule appeared in UBM image. In other words, early cataract was formed in these three eyes. As the ICL with low vault without horizontal dislocation on UBM, the central vault was kept insufficient $(<250 \mu \mathrm{m})$, disappeared, so we classified this situation as a kind of ICL abnormal position.

In contrast, there were excessive vaults $(>750 \mu \mathrm{m})$ in 12 eyes, the maximum value was $1.25 \mathrm{~mm}$. The ICL decentration was noted in varying degrees significantly. Excessive vaults may induce glaucoma, owing to significant angle closure, pupillary block, or pigmentary dispersion. ${ }^{9-12}$ In this study, the elevated IOP occurred in seven eyes with excessive vaults, the mean IOP was $22.12 \pm 3.12 \mathrm{~mm} \mathrm{Hg}$. The chamber angle became narrower; the narrowest point was about only $12^{\circ}$. Iris lost its normal arc shape and became straight because of being ejected by ICL and closed to cornea extremely. Among them, three eyes underwent ICL replacement because of the elevated IOP of more than $25 \mathrm{~mm} \mathrm{Hg}$ for 3 months with glaucoma medications. For the other four eyes after drug treatments, IOPs were controlled in the normal range

Moreover, the ICL-crystalline distances in normal vault (250-750 $\mu \mathrm{m})$ subjects were different in diagonal directions. The distance between the ICL and lens equator was significantly inconsistent Although the subjects had no pain or discomforting symptoms, the ICL decentration was found only in the UBM examination. These subjects were follow-up observed only, did not receive any treatment.

UBM is a contact examination to avoid infection, the postoperative observation time by UBM began 1 month after ICL implantation in this study, so the earliest observed time of ICL anomalies was 1 month postoperatively, and it had already appeared in all these 28 eyes. In the period of observation, the central vaults dropped gradually, but still were in the abnormal range in the insufficient and excessive ones. The ICL

dislocations remained consistent and there were no significant changes in the UBM image.

Several reasons might explain the ICL location changes observed in this study. The current ICL is a flat-plate haptic, single-piece design with a rectangular shape. The haptics of ICL are designed to be located in the sulcus without other assisted-fixed means. The hydrophilic material of ICL is soft, flexible, and biocompatible. Ocular rotations occurring with eye movements may contribute to the ICL decentration.

A limitation of the present study is that the ICL diameter was chosen according to the WTW diameter, and this measurement cannot accurately predict the exact position of the ICL in the sulcus based on this parameter. The size of ICL has a close correlation with the central vault. These reasons may explain how excessive and insufficient vaults were noted in the present study. A prospective study of 61 eyes in 40 Chinese patients who received the V4 design, $1(1.6 \%)$ cataract occurred, and this was because of inadequate vaulting over the crystalline lens from a small ICL. ${ }^{6}$

Insufficient removal of viscoelastic material at the time of the surgery may be another potential contributor to abnormal ICL positioning. The disappearance of the uneven residual viscoelastic material and dynamic interactions between the ICL and the back surface of the iris during accommodation or iris movement ${ }^{1}$ may disproportionately lead the lens to settle posteriorly towards its definitive position near the sulcus.

In conclusion, using panoramic UBM and longer follow-ups, the current study shows the ICL positional change over time. Panoramic UBM, a double-chamber angle display, high-frequency $(50 \mathrm{MHz})$, high-resolution imaging technique, offers cross-sectional and integral images of the anterior segment to a depth of 5-9 mm. ${ }^{13-15}$ Other equipment to inspect the anterior segment, such as Penatacom, Orbscan II, Anterior segment OCT can obtain the anterior segment image also, but under the influence of ICL surface reflection, the image quality is deteriorated, especially in the longitudinal axis, where the ciliary body, lens equator, and ICL footplates cannot be displayed. Panoramic UBM overcomes these limitations, shows the positional relationship between ICL and periphery chamber fabric. UBM is one of the most effective imaging means to observe the ICL position after implantable surgery. This study provides accurate evidence regarding long-term ICL position observation after implantation. Additional studies with direct UBM images of ciliary sulcus with longer followups should be investigated for longitudinal assessment on the behavior to improve safety, anatomical and functional outcomes of ICL implantation.

\section{Summary}

What was known before

- Phakic posterior chamber intraocular lens (ICL) implantation is an effective method for correction of high myopia.

What this study adds

- UBM is one of the most effective imaging means to observe the ICL position after implantable surgery.

\section{Conflict of interest}

The authors declare no conflict of interest. 


\section{Disclaimer}

This work received no specific grant from any funding agency in the public, commercial or not-for-profit sectors.

\section{References}

1 Alfonso JF, Lisa C, Abdelhamid A, Fernandes P, Jorge J, Montés-Micó R. Three-year follow-up of subjective vault following myopic implantable Collamer lens implantation. Graefes Arch Clin Exp Ophthalmol 2010; 248: 1827-1835.

2 Kojima T, Maeda M, Yoshida Y, Ito M, Nakamura T, Hara S et al. Posterior chamber phakic implantable Collamer lens:changes in vault during 1 year. J Refract Surg 2010; 26: 327-332.

3 Choi KH, Chung SE, Chung TY, Chung ES. Ultrasound biomicroscopy for determining Visian implantable contact lens length in phakic IOL implantation. J Refract Surg 2007; 23: 362-367.

4 Guell JL, Morral M, Kook D, Kohnen T. Phakic intraocular lenses part 1: historical overview, current models, selection criteria, and surgical techniques. J Cataract Refract Surg 2010; 36: 1976-1993.

5 Gonvers M, Bornet C, Othenin-Girard P. Implantable contact lens for moderate to high myopia: relationship of vaulting to cataract formation. J Cataract Refract Surg 2003; 29: 918-924.

6 Chang JS, Meau AY. Visian Collamer phakic intraocular lens in high myopic Asian eyes. J Refract Surg 2007; 23: 17-25.
7 Sanders DR. Anterior subcapsular opacities and cataracts 5 years after surgery in the Visian implantable Collamer lens FDA trial. J Refract Surg 2008; 24: 566-570.

8 Bleckmann H, Keuch RJ. Results of cataract extraction after implantable contact lens removal. J Cataract Refract Surg 2005; 31: 2329-2333.

9 Smallman DS, Probst L, Rafuse PE. Pupillary block glaucoma secondary to posterior chamber phakic intraocular lens implantation for high myopia. J Cataract Refract Surg 2004; 30: 905-907.

10 Vetter JM, Tehrani M, Dick HB. Surgical management of acute angle-closure glaucoma after toric implantable contact lens implantation. J Cataract Refract Surg 2006; 32: 1065-1067.

11 Bylsma SS, Zalta AH, Foley E, Osher RH. Phakic posterior chamber intraocular lens pupillary block. J Cataract Refract Surg 2002; 28: 2222-2228.

12 Chung TY, Park SC, Lee MO, Ahn K, Chung ES. Changes in iridocorneal angle structure and trabecular pigmentation with STAAR implantable Collamer lens during 2 years. J Refract Surg 2009; 25: 251-258.

13 Pavlin CJ, Harasiewicz K, Sherar MD, Foster FS. Clinical use of ultrasound biomicroscopy. Ophthalmology 1991; 98: 287-295.

14 Deramo VA, Shah GK, Baumal CR, Fineman MS, Corrêa ZM, Benson WE et al. Ultrasound biomicroscopy as a tool for detecting and localizing occult foreign bodies after ocular trauma. Ophthalmology 1999; 106: 301-305.

15 Looi ALG, Gazzard G, Tan DTH. Surgical exploration minimised by ultrasound biomicroscopy localisation of intraocular foreign body. Eye (Lond) 2001; 15: 234-235. 